\title{
The Drosophila hedgehog gene is expressed specifically in posterior compartment cells and is a target of engrailed regulation
}

\author{
Tetsuya Tabata, Suzanne Eaton, and Thomas B. Kornberg \\ Department of Biochemistry and Biophysics, University of California at San Francisco, San Francisco, California 94143 USA
}

\begin{abstract}
cDNAs were isolated that represent transcripts of the Drosophila segment polarity gene, hedgehog (hh). Sequence analysis reveals a motif characteristic of a transmembrane domain, suggesting that the hh protein is membrane-associated. $h$ h expression in epidermal cells is confined to the posterior compartments and coincides precisely with that of engrailed (en). Despite the similar patterns of expression in the cellular blastoderm, $h \boldsymbol{h}$ expression is independent of $e n$, but $h \boldsymbol{h}$ expression becomes sensitive to and dependent on $e n$ during the extended germ band stage. The ectopic expression of $h$ h that is normally induced in patched (ptc) mutant embryos does not appear in ptc en double mutants. We discuss these findings in terms of the relationship between $e n$ and $h h$, and the role of the hh function.
\end{abstract}

[Key Words: Drosophila; hedgehog; engrailed]

Received September 24, 1992; revised version accepted November 2, 1992

The segmented epidermis of Drosophila is composed of two distinct populations of cells: the anterior and posterior compartments. These compartments appear to be the development units in which growth, polarity, and pattern are regulated (Lawrence and Morata 1976). They are also clonally isolated areas whose intimate juxtaposition belies their separatist behavior, as their constituent cells confront each other at their shared borders but do not intermingle during growth and development (Garcia-Bellido et al. 1973).

Our understanding of the properties that distinguish the cells of the two compartments and of the mechanisms that endow the compartment borders with their special functions is rudimentary. Even if genetically endowed with the capacity to overgrow their neighbors, epidermal cells generate normal patterns and do not grow beyond their compartment borders (Garcia-Bellido et al. 1973/. Their growth is not limited by obvious physical barriers, because in general, compartment borders do not coincide with morphological structures. Rather, their developmental commitments and growth limitations are under the control of the engrailed (en) gene (Morata and Lawrence 1975). In the epidermis, en is expressed only by the posterior compartment cells (Kornberg et al. 1985; Hama et al. 1990), and its function is essential to the processes that generate pattern in the posterior compartments and define the compartment borders as limits to growth (Lawrence and Morata 1976).

The en gene encodes a homeo domain-containing nuclear protein (DiNardo et al. 1985; Poole et al. 1985) which probably acts as a transcription factor to regulate the expression of other genes (Jaynes and O'Farrell 1991). Presumably, it is the combined action of these target genes that define the unique characteristics of posterior compartment cells. Several putative targets of en regulation have been identified. Cubitus interruptus-Dominant $(c i-D)$ is expressed exclusively in anterior compartment cells, and its repression in posterior compartment cells depends on en function (Eaton and Kornberg 1990). The sequence of the ci-D protein is similar to that of the human GLI proteins. Because GLI proteins are DNAbinding proteins localized in nuclei (Kinzler and Vogelstien 1990), it seems likely that the ci-D protein might regulate transcription in some manner /Orenic et al. 1990). Another potential transcription factor target of en regulation is polyhomeotic, one of the Polycomb group of genes that are required to repress the expression of many homeotic genes (Jürgens 1985). polyhomeotic expression in the early embryo depends on en function and is largely absent in en mutants (F. Maschat, N. Serrano, C. Demeret, J.M. Dura, H.W. Brock, and T.B. Kornberg, in prep.). In this paper, we present evidence for the identification of another target of en regulation, hedgehog (hh).

hh has been classified as one of the segment polarity group of zygotically active segmentation genes. The genes of the segment polarity group regulate spatial patterning within each segment (Nüsslein-Volhard and Wieschaus 1980). Putative functions of the proteins encoded by the segment polarity genes have been deduced 
from the sequences of their respective transcripts. Such functions include transcription factors [e.g., en (Jaynes and O'Farrell 1988), gooseberry (Bopp et al. 1986), and ci-D (Orenic et al. 1990; Eaton and Kornberg 1990)], a signaling molecule [wingless (wg; Baker 1987; Rijsewijk et al. 1987)], a membrane-bound receptor [patched (ptc; Nakano et al. 1989; Hooper and Scott 1989)l, a protein kinase [fused (Preat et al. 1990)], and a plakoglobin/ $\beta$ catenin-like molecule [armadillo (Peifer and Wieschaus 1990; McCrea et al. 1991)]. Segment polarity gene proteins whose function are less well understood include dishevelled (dsh), naked (nkd), and hh.

Most $h$ mutants are embryonic lethal, and mutants secrete a ventral cuticle that has little segmental patterning or anterior/posterior polarity (Nüsslein-Volhard and Wieschaus 1980; Mohler 1988). Genetic interactions with other segmentation genes have led to the suggestion that $h h$ is involved in cell-cell communication and that it helps to regulate $w g$ through its interactions with the ptc protein (Ingham et al. 1991). Adult cuticular phenotypes observed in mosaic adults indicate partially nonautonomous effects that are mostly associated with structures in posterior compartments (Mohler 1988).

Determination of the sequence of the putative hh protein now reveals that the hh protein contains sequences common to membrane-associated proteins. Interestingly, in trunk region of embryos and in imaginal discs, $h h$ is expressed exclusively in posterior compartment cells; and in these embryos, $h h$ is positively regulated by en. These results are in agreement with and confirm the proposed partial sequence of the hh protein that was deduced from genomic and partial cDNA sequencing (Mohler and Vani 1992). In view of the close regulatory and spatial relationship between $h h$ and $e n$, we suggest that the role of $h h$ in posterior compartments is to identify a cell that expresses en protein and to communicate this information to neighboring cells.

\section{Results \\ Isolation and analysis of hh cDNAs}

A previous report described an enhancer trap screen designed to identify genes whose expression correlates with the anterior/posterior compartment subdivisions of imaginal discs (Eaton and Kornberg 1990). We have extended this screen and have found that transposon insertions at four cytological locations (48A, 89A, 94D/E, and 101F) were associated with compartment-specific expression. Enhancer trap strains with insertions at 89A and $101 \mathrm{~F}$ had $l a c Z$ expression limited to anterior compartment cells; 48A and 94D/E strains had lac $Z$ expression limited to posterior compartment cells. $101 \mathrm{~F}$ is the site of the ci-D gene (Eaton and Kornberg 1990; Orenic et al. 1990), and the $\beta$-galactosidase activity in the $101 \mathrm{~F}$ enhancer trap strain accurately reflects the patterns of $c i-D$ expression. Strain $89 \mathrm{~A}$ is apparently a hot spot for enhancer trap insertions (eight examples were isolated); the relevant transcription unit has been identified, but its function has yet to be established (S. Eaton and T.B. Kornberg, unpubl.). Strain 48A is the site of the en gene (Kornberg 1981); 94D/E is at or near the $h h$ gene.

Two 94A/E lines were identified. Line A937A carries a P element marked with white ${ }^{+}$; P2023 carries a P element marked with rosy ${ }^{+}$. Their patterns of $\beta$-galactosidase activity in imaginal discs were indistinguishable, although $\beta$-galactosidase activity in A937A embryos was not detectable. Their sites of insertion are within $1 \mathrm{~kb}$ (Fig. 1A). The A937A strain is homozygous viable and has no apparent phenotype. One hundred white ${ }^{+}$revertants were isolated after A937A flies were exposed to transposase provided by the D2-3 P element (Robertson et al. 1988). Three were lethal; and as indicated by Southern analysis, they had small deletions at the former site of insertion. The P2023 strain is lethal, as were all of $\sim 100 \Delta 2$-3-induced $\mathrm{ry}^{-}$revertants. The A937A lines represent a single complementation group and complemented P2023. All of the P2023 lines as well as the A937A lethal revertants complemented hh mutants. These observations suggest that the lethality of the P2023 group is not associated with the insertion at $94 \mathrm{D} / \mathrm{E}$ and that neither of the lethal complementation groups represents $h h$. The proximity of the A937A insertion to unkempt (unk) (Fig. 1A) suggests, instead, that the lethality of the imprecisely excised revertants was the result of loss of unk function (Mohler et al. 1992), although no crosses to unk mutants were performed to test this possibility.

To identify the gene whose posterior compartmentspecific expression is reflected in the A937A and P2023 enhancer trap lines, genomic DNA from the region of the insertions was analyzed. Approximately $58 \mathrm{~kb}$ of genomic DNA from the 94D/E region was isolated in five $\lambda$ phage. Northern analysis, "reverse Northern" analysis, and in situ hybridization to embryos revealed several genomic regions that are transcribed in embryos (Fig. 1A,B). Comparisons with unpublished sequence and restriction maps of $\mathrm{J}$. Mohler (pers. comm.) indicated that the transcribed region closest to the transposon insertions is unk, and that $h h$ is $\sim 15 \mathrm{~kb}$ farther away.

Northern analysis of embryonic and imaginal disc RNA using a probe from the $h h$ region (probe $B$; Fig. 1A) detected a single poly(A)-containing RNA of $\sim 2.4 \mathrm{~kb}$. In situ hybridization with the same probe revealed that transcripts derived from the $h h$ region were arrayed in zebra-stripe patterns (Fig. 2), which are indistinguishable from the patterns of $\beta$-galactosidase activity in the P2023 enhancer trap strain (see Figs. 2E and 3). Transcripts from the unk region were also detected in embryos; they were ubiquitous and abundant (not shown). In situ hybridization to whole-mount embryos with probes from $58 \mathrm{~kb}$ of genomic DNA revealed only the unk and $h$ transcripts. We conclude that in the $58-\mathrm{kb}$ portion of $94 \mathrm{D} / \mathrm{E}$ that we isolated, only the unk and $h h$ genes are transcribed in embryos. Furthermore, P2023 responds to the regulatory elements that control $h$ h expression, despite the distance of $>15 \mathrm{~kb}$ and the interposed unk gene.

cDNA representatives of the $h h$ transcripts were isolated and sequenced (Fig. 4). The longest cDNA has 2.3 

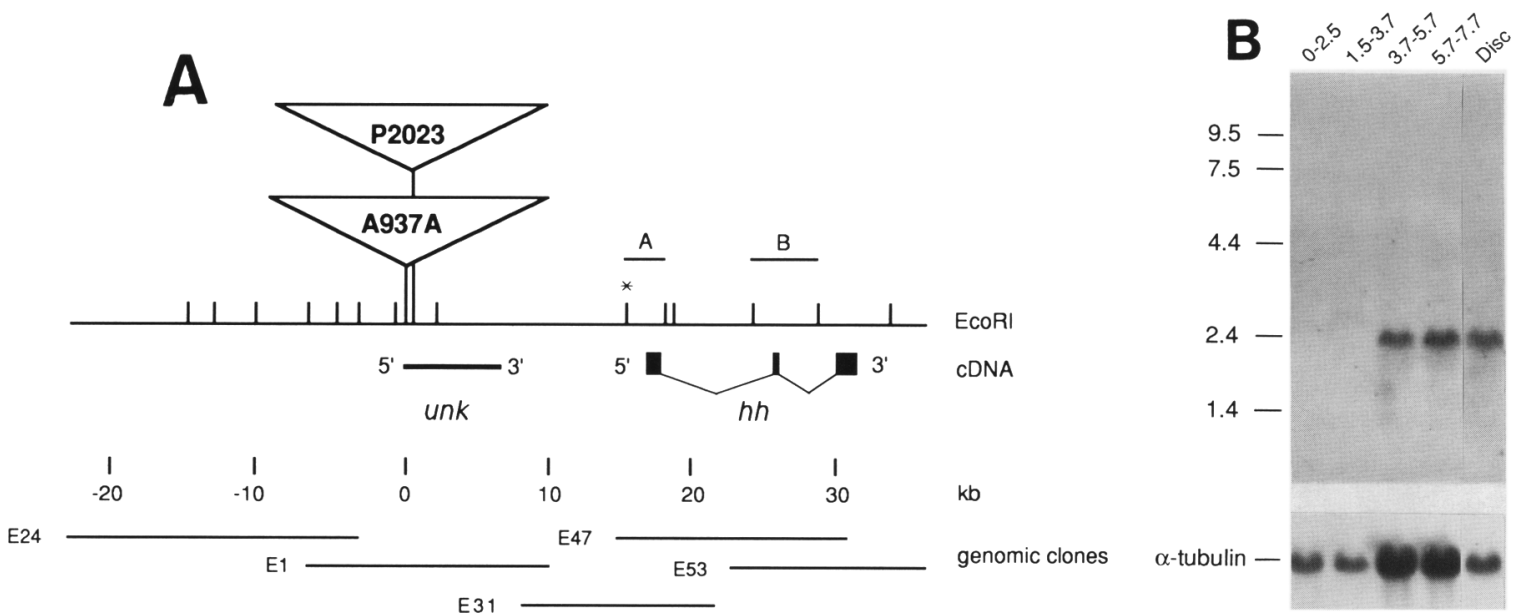

Figure 1. The $h h$ region. $(A)$ Indicated above the solid horizontal line representing the chromosomal DNA are the positions of integration of two P-element enhancer trap strains, A937A and P2023; the relative positions of the genomic inserts in five $\lambda$ phage $(\lambda \mathrm{E} 24, \lambda \mathrm{E}, \lambda \mathrm{E} 31, \lambda \mathrm{E} 47$, and $\lambda \mathrm{E} 53)$; the locations and direction of transcription of the unk and $h h$ genes; and the approximate location of probes A and B used to screen the cDNA library. The colinearity of the third exon of $h h$ and of the genomic DNA was not mapped rigorously. The EcoRI site ( $\left.{ }^{*}\right)$ was found in only one of several phage clones covering this region. Coordinates are in kb and orientation is centromere to the right. $(B)$ Northern analysis with probe B from the $h h$ region detected a single species of oligo(dT)-selected RNA in cellular blastoderm embryos, germ-band-extended embryos, and imaginal discs. Lower panels show the same lanes hybridized with an $\alpha$-tubulin probe. Times indicated reflect hours after egg laying; molecular weight estimates were with reference to RNA standards.

$\mathrm{kb}$ and contains a putative open reading frame capable of encoding a $52-\mathrm{kD}, 471$-residue protein. Near the amino terminus of this putative protein is a hydrophobic sequence of 19 amino acids (residues 63-81) that is similar to the membrane-spanning regions of other proteins e.g., residues 182-201 of Escherchia coli CvaB inner membrane protein; Gilson et al. 1990). These results are consistent with the partial sequence obtained by Mohler and Vani (1992) and suggest that the hh protein may be membrane associated.

\section{Patterns of hh expression}

Mohler and Vani (1992) reported that hh expression in embryos is localized in posterior compartment cells. To verify this observation, we compared $h h$ and en expression in two ways. We compared embryos that had been subjected to in situ hybridization using probes for $h h$ RNA or en RNA (Fig. 2), and we prepared embryos and imaginal discs so that $h h$ and en expression could be monitored simultaneously (Fig. 5). Our observations largely support the conclusions of Mohler and Vani (1992) and, in early embryos, reveal some differences between the distributions of $h h$ and $e n$ RNA.

At the cellular blastoderm stage, $h h$ transcripts were localized predominantly in a single stripe at $-75 \%$ egg length, although additional hybridization was apparent at the anterior tip and along the ventral side (Fig. 2A). Cellular blastoderm embryos initially expressed en RNA in a single stripe (stripe 2 ) at $\sim 60 \%$ egg length (Fig. $2 \mathrm{~B}$, a slightly older embryo; see also Karr et al. 1989). With the commencement of gastrulation (Fig. 2C,D), hh and en transcripts formed 14 single-cell-wide stripes between $30 \%$ and $65 \%$ egg length. Only $h h$ expressed RNA in a block of cells at the anterior end and in wide stripes at $10 \%$ and $75 \%$ egg length. The 14 stripes of en RNA do not appear simultaneously but appear in a characteristic order-even-numbered ones before odd-numbered ones and anterior ones before posterior ones (Weir and Kornberg 1985). The 14 single-cell-wide stripes of hh RNA follow a similar progression (not shown).

During subsequent stages of embryogenesis, the patterns of $h h$ and en RNA were apparently identical, except in the terminal regions (Fig. 2E-H). hh RNA was also detected in the foregut (later resolving into pharynx and esophagus expression), hindgut, and salivary gland (Fig. 2I,J). To examine the correspondence of $h h$ and en expression more precisely, embryos were examined that had been stained with anti-en antibody and had been probed for hh RNA. No cells expressing only hh or en were observed in the 14 segmental stripes (Fig. 5B). However, in the regions of the head that express only one of the two genes, cells labeled with only one of the probes could be detected (Fig. 5A-D). We conclude that in the segmented trunk of the embryo, $h h$ is coexpressed with en in the cells of the posterior compartments.

Expression of $h h$ in imaginal discs is also localized to the posterior compartments. $\beta$-Galactosidase activity in discs dissected from P2023 third-instar larvae (Fig. 3A) revealed patterns similar to en expression (Hama et al. 1990). The distributions of lacZ RNA and en protein in wing discs was indistinguishable (not shown).

\section{hh expression in mutant embryos}

To identify genetic factors that regulate $h h$ expression, patterns of $h h$ transcripts were compared with patterns of en protein in mutant embryos lacking zygotically ac- 
Figure 2. Comparison between patterns $h h$ and en expression in embryos. Whole mounts of embryos after in situ hybridization with either a probe for $h h$ RNA $\{A, C, E, G, I, I)$ or for en RNA $(B, D, F, H) .(A, B)$ stage $5 ;(C, D)$ stage $6 ;(E, F)$ stage $11 ;(G-I)$ stage $13 ;(J)$ stage 16 . Small arrowheads in $A$ and $C$ mark regions of $h$ h-specific expression. Large arrowheads in $A-D$ mark stripe 2 of the $h h$ and en patterns. Expression in the salivary gland primordium is marked with arrowheads in $I$. In this and all subsequent figures, embryos are oriented anterior to the left and dorsal up, unless indicated otherwise. Nomarski optics were used.
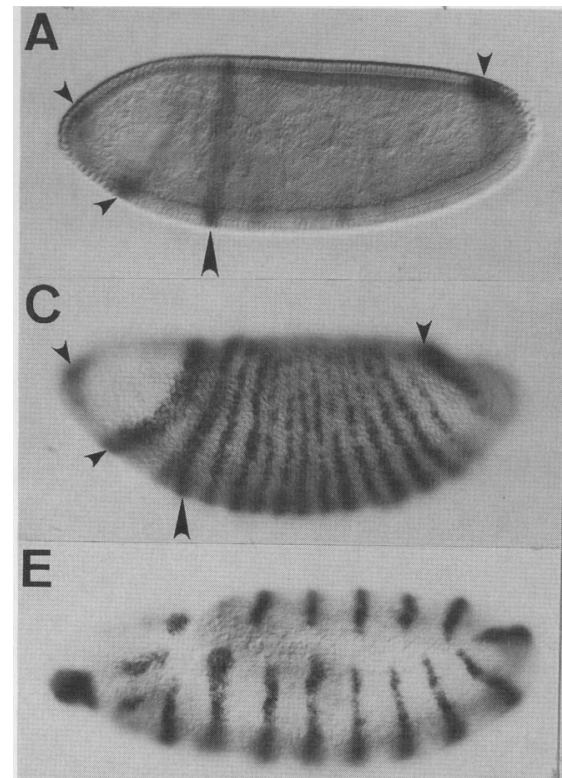

$F$
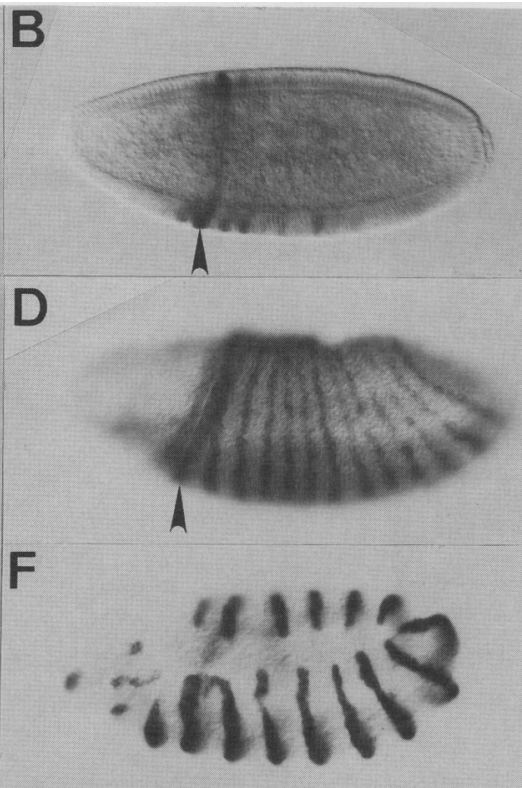

G

H
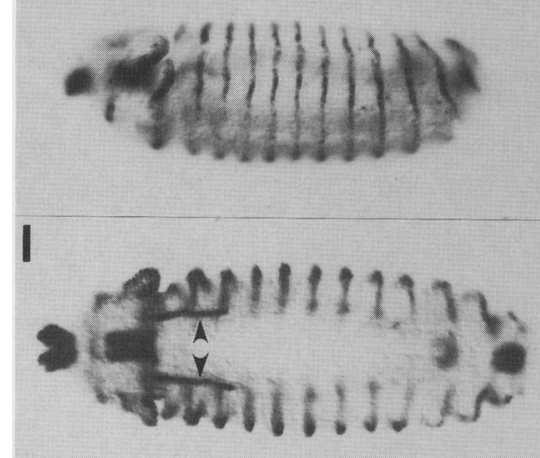

J
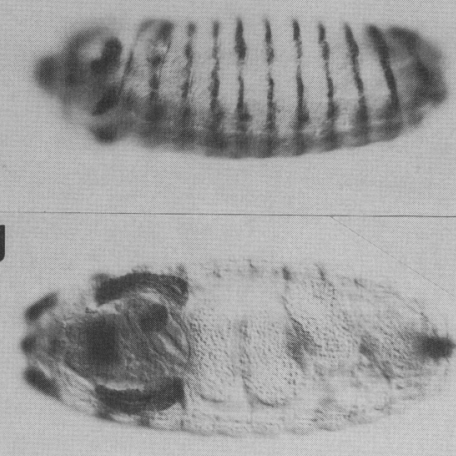

tive segmentation genes. In agreement with previous findings, we found that in the segmented trunk region of embryos, the patterns of en protein are altered by mutations in both pair-rule and segment polarity genes (Martinez-Arias et al. 1988). fushi tarazu ( $f t z)$ mutants lacked alternate en stripes (Fig. 6B); wg mutants failed to sustain en expression (Fig. 6D); nkd mutants had abnormally wide stripes (Fig. 6F); and ptc mutants had ectopic stripes (Fig. $6 \mathrm{H}$ ). Patterns of $h$ h RNA visualized by in situ hybridization were virtually indistinguishable from the patterns of en protein. ftz embryos lacked even-numbered $h$ h stripes (Fig. 6A). Before stage 10, wg embryos had normal hh expression (not shown); however, hh expression in the segmented trunk region began to decay at stage 10 (Fig. 6C) and disappeared by stage 11 (not shown). hh RNA in the maxillary and labial segments was unaffected by loss of wg function. nkd mutants had stripes that were abnormally wide in stage 11 (Fig. 6E) and in older embryos (not shown). Ectopic $h$ h RNA appeared in stage 11 ptc embryos (Fig. 6G) and persisted at least through stage 13 (not shown).

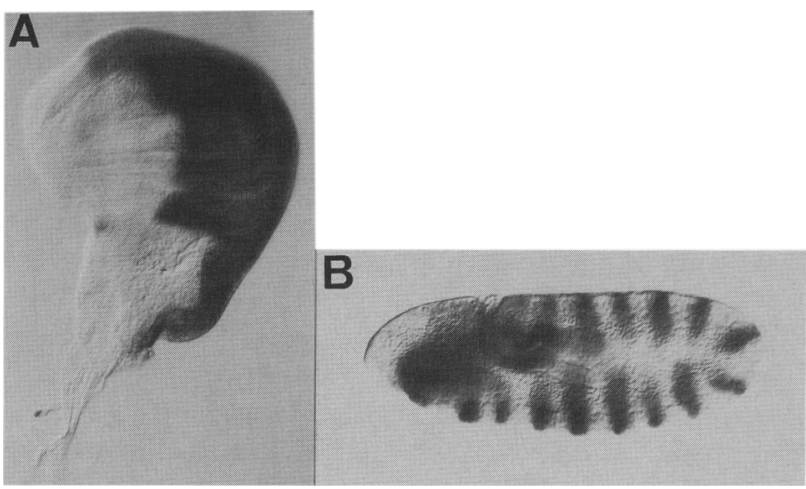

Figure 3. Patterns of $\beta$-galactosidase activity in the P2023 enhancer trap strain. $(A)$ Wing imaginal disc from a third-instar larva stained with $\mathrm{X}$-gal. The region with $\beta$-galactosidase activity is the posterior compartment, and its shape is indistinguishable from the region of en expression. $(B) \mathrm{X}$-gal staining of an extended germ-band stage embryo (stage 11) reveals patterns identical to patterns of $h$ h RNA (see Fig. 2E). Nomarski optics were used. 


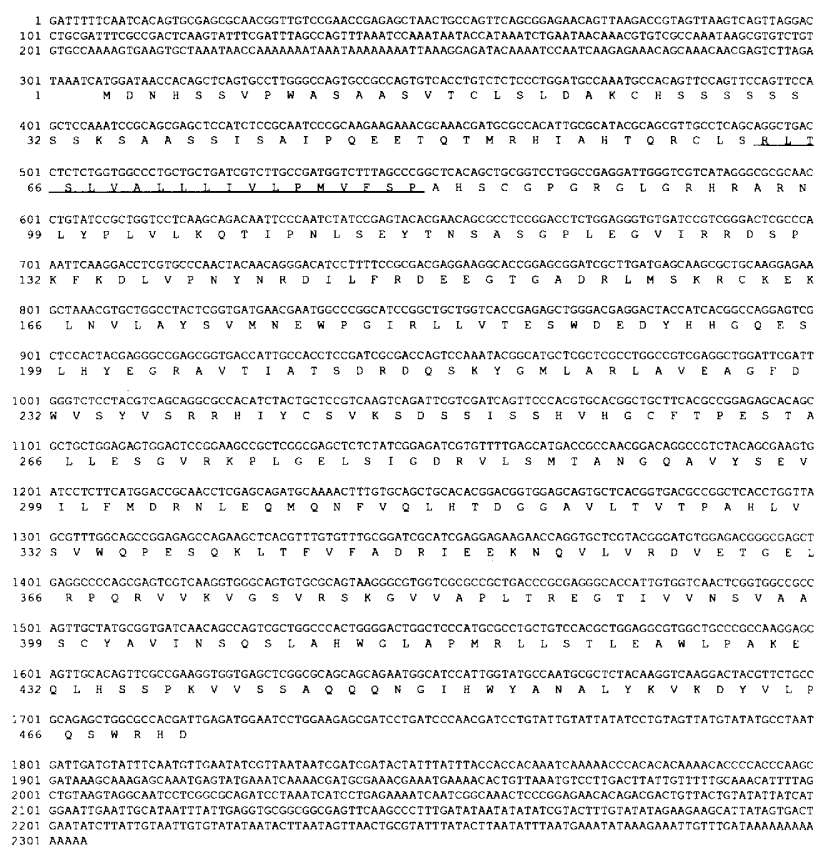

Figure 4. Sequence of the $h$ cDNA clone chh46. Translation of the 1413-bp open reading frame starting from the ATG at position 307 is also shown. In the regions upstream and downstream of this large open reading frame, all three frames are closed. The putative transmembrane region is underlined.

Before stage 10, no change in hh RNA expression was detected in en embryos (not shown). However, hh RNA stripes in the segmented trunk region did not sustain their intensity or extent in early stage 11 embryos (Fig. 6I) or in subsequent stages. In contrast to the effects on the segmental stripes, expression in the foregut and hindgut was unchanged. Dependence on en function for segmental stripe expression was also observed in ptc en embryos. In these double mutant embryos, the extent and intensity of the stripes diminished after stage 10 . Interestingly, no ectopic stripes of $h h$ RNA appeared in stage 11 double mutant embryos (Fig. 6J). We conclude that after stage 11, embryos require en function both for maintenance of $h h$ expression in the segmental stripes (Fig. 6I) and for activation in the ectopic locations.

In addition to the lack of ectopic stripes in double mutant embryos, the pattern of denticles on the ventral cuticle of ptc en embryos was also not affected as severely as either ptc or en single mutant embryos (Fig. 7; Hidalgo 1991). We conclude that ptc mutations can partially suppress the en phenotype and, conversely, that en mutations can partially suppress the ptc phenotype. Moreover, the ectopic furrows that form at the sites of ectopic en expression in ptc mutants also form in the double mutant embryos (Fig. 6J), indicating that they do not reflect the formation of ectopic en-dependent segment boundaries.

Ingham et al. (1991) demonstrated that the stripes of $w g$ expression in stage 11 embryos broaden anteriorly in ptc mutants and in ptc; hh mutants. We confirmed their observations (not shown) and find that ptc en mutants express $w g$ in similarly broader stripes (Fig. 6K,L). Apparently, in the absence of $p t c$ function, wg expression, which is normally en-dependent, no longer requires en.

\section{hh responds to ectopic en expression}

The apparent dependence of $h h$ on en function in stage 11 embryos, and the coexpression of the two genes in posterior compartment cells during subsequent development suggest that en assumes control of $h h$ expression during mid-embryogenesis. Unfortunately, it is difficult to test this hypothesis with en mutants, because most en mutants are embryonic lethals, and viable combinations have only marginal effects on en expression (Condie and Brower 1989|.

An alternative approach is to induce en in ectopic locations and to monitor the effects on $h h$. We induced ectopic en expression with a strain (hs-en3) carrying a $P$ element with a fusion gene consisting of the en cDNA sequence and an $h s p 70$ promoter. hs-en 3 was subjected to heat shock during embryogenesis; and after appropriate incubation, hh expression was detected by in situ hybridization. It has been shown previously that a pulse of heat shock during the gastrulation period increases the width of stripes of en-expressing cells during later germ band elongation stages (Heemskerk et al. 1991) and subsequently produces en mutant phenocopies at the end of embryogenesis (Poole and Kornberg 1988). hh expression is altered in a similar manner after heat shock of hsen3 (Fig. 7C), as the bands of en-expressing cells expand along the ventral midline at stage 11 (Fig. 7D). To variable extents, both hh RNA and en protein are also observed in other ectopic locations in the anterior regions of the segments. We conclude that under these conditions, $h h$ is positively regulated by en during the later stages of embryogenesis.

\section{Discussion}

$h h$ is a segment polarity gene that encodes an essential function in both embryos and adults. Mutant $h h$ embryos die after secreting a cuticle with severely perturbed ventral denticle belts (Nüsslein-Volhard and Wieschaus 1980); mosaic patches of hh cells generate morphologically abnormal structures in the adult cuticle (Mohler 1988). Our studies and those of Mohler (Mohler and Vani 1992) demonstrate that patterns of $h$ h RNA expression correlate well with these phenotypes and provide a general framework with which to consider the functional role of $h h$.

Animals that are homozygous for strong $h h$ alleles develop to $\sim 40 \%$ the length of wild-type larvae and have a ventral cuticle with unusually small denticle-free regions, abnormally patterned denticle belts, and poorly defined separations between adjacent denticle belts (Mohler 1988). These characteristics are common to mutants in other segment polarity genes as well. For instance, en mutant embryos are unusually short (approx- 
Figure 5. Comparisons of patterns $h h$ and en expression. (A) Distribution of $h$ RNA (blue) and en protein (brown) in a stage 11 wild-type embryo revealed by in situ hybridization and immunohistochemical detection. With the exception of the cells indicated by the anterior white arrow, $h h$ and en expression is completely coincident, and cells that are solely labeled with blue or brown pigments were not observed. $(B-E)$ Higher magnification views of the region in $A$ indicated by white arrowheads. $(B)$ View of the area in $A$ indicated by the anterior arrow. $(C)$ View of an embryo of similar stage as $A$ probed only for $h h$ RNA. Note the peripheral, non-nuclear staining. $(D)$ View of an embryo of similar stage as $A$ probed only with anti-en antibody. Note the nuclear staining. $|E\rangle$ View of the stripes in (A) indicated by the posterior arrow. Note the presence of stain in both nuclear and nonnuclear areas of the cells in the stripes. Nomarski optics were used.

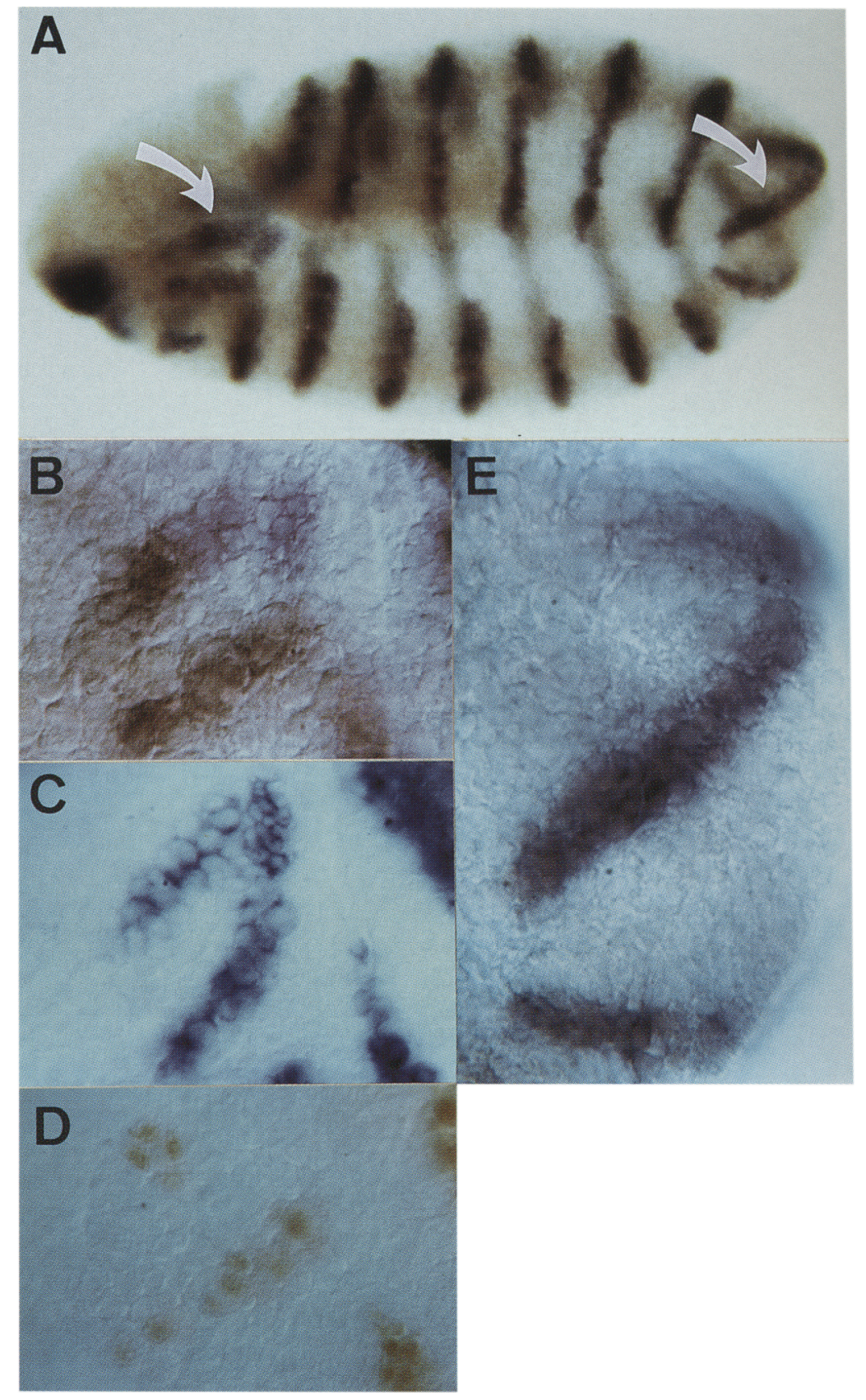

imately two-thirds normal length), the naked cuticle between their ventral denticle belts is reduced, and their distorted denticle belts fuse together (Kornberg 1981).

The imaginal phenotypes of $h h$ and en are also similar. Homozygous mutant $h h$ and $e n$ cells are viable in mosaic flies, and develop normally in the anterior compartments of the antennae, legs, wings, and notum. In contrast, mutant $h h$ and $e n$ cells in posterior compartments develop abnormally, disturbing the morphology or reducing the size of the organs in which they grow (Kornberg 1981; Lawrence and Struhl 1982; Mohler 1988). Abnormalities associated with en cells were found exclu- sively with clones in the posterior compartments of the epidermis. Because these studies characterized both hairand bristle-producing en cells, the size and extent of the mutant clones could be precisely determined. A similar analysis of $h h$ mutant cells was more limited in its resolution, because the chromosomal location of $h h$ made it possible to mark only bristle-generating cells (Mohler 1988). As a consequence, the role of hh could only be examined in areas of the cuticle-containing bristles. Nevertheless, with few exceptions, clones with abnormal morphology were associated with mutant posterior compartment cells, indicating that the role of $h h$, like 


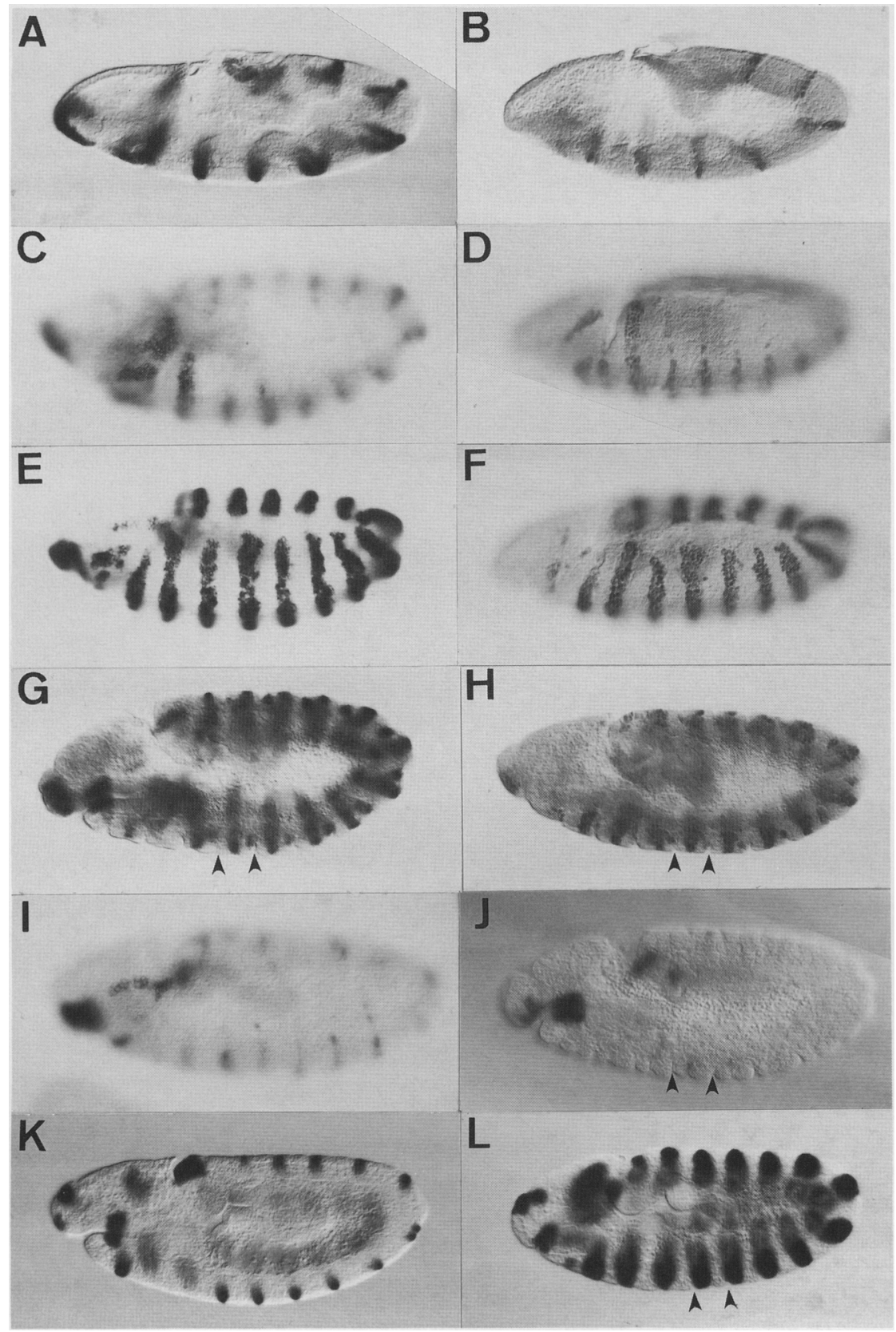

Figure 6. Altered segment polarity gene expression in mutant embryos. $A-J$ compare $h h$ and en expression in $f t z, w g$, en, $n k d, p t c$, and ptc en mutant embryos. $K$ and $L$ compare $w g$ expression in wild-type and ptc en mutant embryos. Whole mounts revealing $h h$ RNA $(A, C, E, G, I, J)$, en protein $(B, D, F, H)$, or wg RNA $(K, L)$ in stage $8 \mathrm{ftz}(A, B)$, stage $10 \mathrm{wg}(C, D)$, stage $11 \mathrm{nkd}(E, F)$, stage $11 \mathrm{ptc}(G, H)$, stage $11 \mathrm{en}$ $(I)$, stage 11 wild-type $|K|$, and stage 11 ptc en $(I, L)$ mutant embryos are shown. Arrowheads in $G, H, J$, and $L$ indicate ectopic furrows that are characteristic of ptc mutants. Nomarski optics were used. en, is posterior compartment specific. The only notable exceptions were in regions that either lacked bristles (e.g., the posterior scutellum) or in which the precise location of the anterior/posterior compartment border is uncertain (e.g., in the ocellar region, the arista and second antennal segment, and the genitalia).

Mohler and Vani (1992) demonstrated that the pattern of $h h$ expression in embryos is in the region of the posterior compartments, and we have confirmed and extended these observations. Embryos labeled to illuminate both $h h$ mRNA and en protein reveal that expression of $h h$ and en correlate precisely in the posterior compartments of the thoracic and abdominal segments.
In contrast, their expression patterns differ in the head and terminalia, where anterior and posterior compartments do not form until later during larval development, and in the salivary glands and nervous system, where developmental compartments do not apparently form. In imaginal discs, $h$ h expression was also shown to colocalize with the en-expressing cells of the posterior compartments. The requirement for $h h$ in the epidermal posterior compartments is therefore consistent with its pattern of expression. In the posterior compartments, $h h$, like en, is a posterior compartment-specific gene.

How are the compartment-specific patterns of $h h$ and en expression controlled? Because en is likely to be a 
Figure 7. Ventral cuticular phenotypes of en and ptc embryos. Panels compare wildtype, en, ptc, and ptc en embryos after dechorionation, devitellinization, and mounting in Hoyer's aqueous mountant. Lower panels are higher magnification views of A6 and A7. Orientation is anterior up. Phase-contrast optics were used.
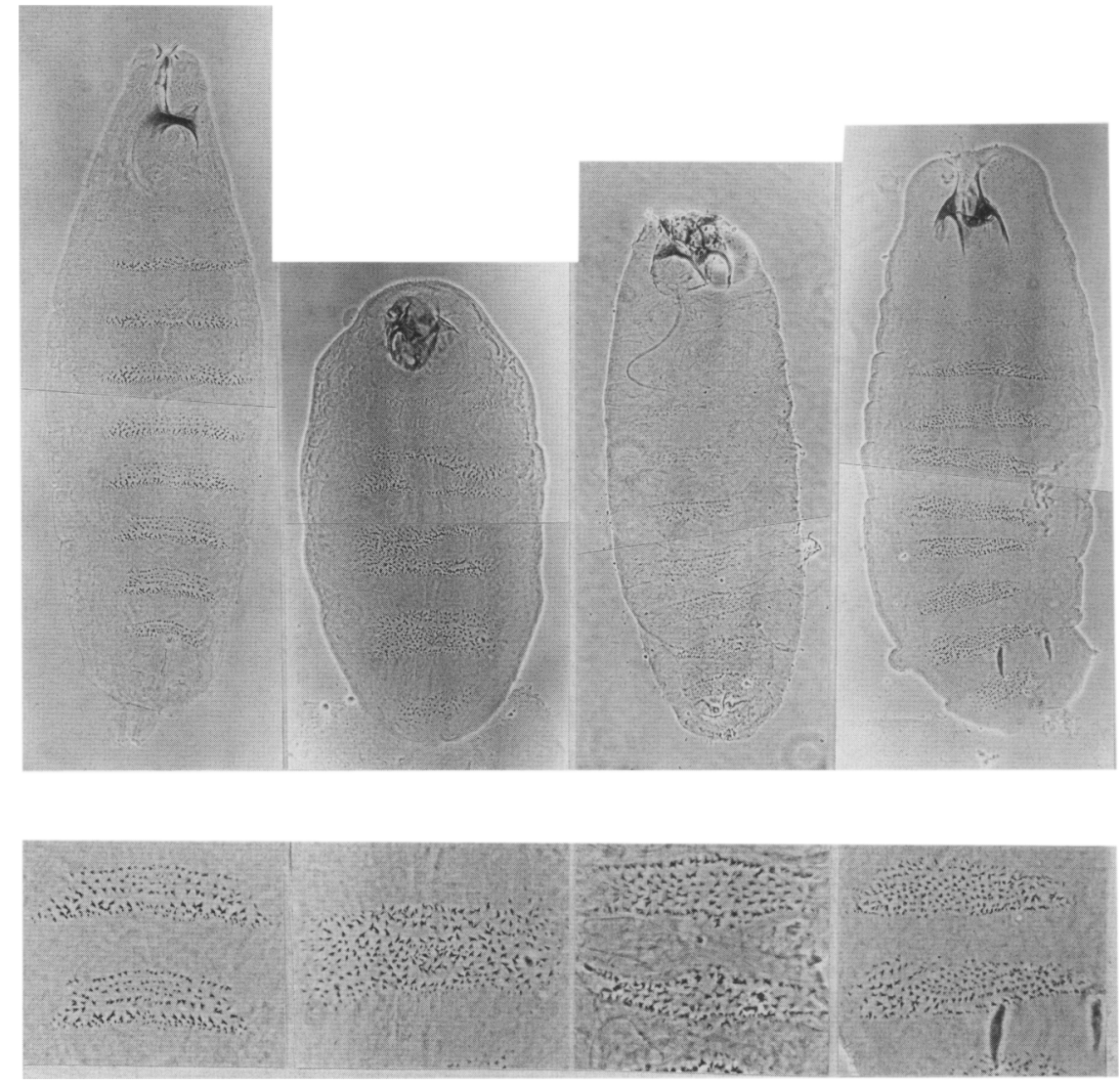

transcription factor, it is a candidate to positively regulate $h h$. Previous studies, for instance, have shown that the anterior compartment-specific pattern of ci-D expression involves negative regulation by $e n$ in the posterior compartments (Eaton and Kornberg 1990). However, $h h$ is activated normally at the cellular blastoderm stage in en mutant embryos, and en is activated normally in $h h$ mutant embryos. $h h$ and $e n$ expression at these early embryonic stages is independently controlled. Because the expression of both genes is affected similarly in mutants that lack $f t z$ (Fig. 6) or even-skipped (not shown), it seems that their activation is accomplished directly or indirectly by pair-rule segmentation genes.

During the germ-band elongation stages, the mechanisms that regulate $h h$ and en expression change. Both genes responded similarly to ptc /gaining an ectopic stripe of expression in each segmental unit) and to $n k d$ (adding an extra row of expressing cells to each stripe) mutant backgrounds (Fig. 6; Martinez-Arias et al. 1988). In addition, $h$ h expression became dependent on en function at stage 11 (Fig. 6I,J), and $h h$ was expressed in ectopic locations after heat shock induction of en (Fig, 8). These observations suggest that $h h$ is a target of en positive regulation at germ-band elongation. Furthermore, the similar phenotypes of ptc en (Fig. 7) and ptc; hh (Ingham et al. 1991) double mutant embryos suggest that en and $h h$ act through a common pathway with respect to $p t c$. One possible explanation for these observations is provided in a model proposed previously by Ingham (Ingham et al. 1991), whereby $h h$ acts as a ligand for the ptc protein. If binding of the hh ligand to the ptc protein receptor reverses an indirect antagonistic effect that ptc protein would otherwise have on wg transcription, then the normal role of hh protein would be to maintain wg transcription in the cells adjacent to en-expressing cells. This model provides an explanation for ptc en double mutant phenotypes. Assuming that $h h$ is regulated by en, $p t c$ mutants would uncouple $w g$ expression from en and $h h$ activities and would thereby suppress the en and $h h$ phenotypes by relieving $w g$ from $p t c$ repression (Fig. 7). Our observation that wg expression persists in ptc en embryos is consistent with this proposal (Fig. 6L).

It bears noting that despite their similar patterns of expression in germ-band-stage embryos and in imaginal discs ( $p t c$ is expressed at the compartment borders and both $e n$ and $h h$ are expressed in all posterior compartment cells), the roles of en, $h h$, and ptc must differ in embryos and discs if the interactions between $h h$ and $p t c$ are direct and involve $w g$. $w g$ is not expressed along the anterior/posterior compartment border in imaginal discs as it is in embryos. Furthermore, although a finer resolution analysis will be required to firmly establish the 


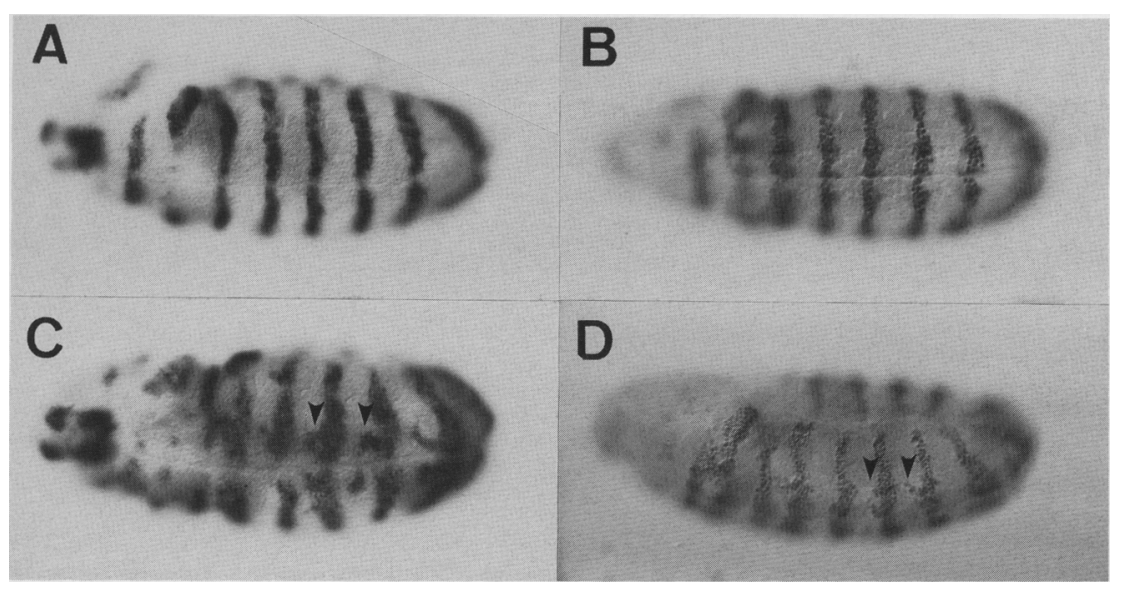

Figure 8. Ectopic expression of en induces ectopic $h$ h expression. hsen3, a strain that can express en under the control of the $h s p 70$ promoter, was subjected to a pulse of heat shock at $37^{\circ} \mathrm{C}$ as described in Materials and methods. $h h$ RNA $(A, C)$ and $e n$ protein $(B, D)$ in stage 11 embryos that either had $\{C, D\}$ or had not $|A, B|$ been heat shocked during the gastrulation period. Note that the stripes are wider after heat shock. Arrowheads in $C$ and $D$ indicate the variable patches of ectopic expression. Nomarski optics were used.

point, the phenotype of $h h$ mutant clones suggests that $h h$ has an essential role among the posterior compartment cells that do not directly contact the compartment border. This role must be independent of both ptc and $w g$.

What is the role of $h h$ in posterior compartments? Although we can only postulate what its role might be at this time, the possibility that hh protein is a cell-surface protein suggests that it could serve to mark the surface of all posterior compartment cells. There it could signal an identity as a posterior compartment, en-expressing cell to neighboring cells. In doing so, it could play several roles. Because anterior compartment cells dö not express hh protein on their surface, hh function could be involved in maintaining the physical separation between anterior and posterior cells, if cells that express hh preferentially associate with one another. Such a mechanism based on differential cell affinities was proposed by Lawrence and Morata (1976) to explain the lineage restrictions of developmental compartments. Another possibility is that posterior compartment cells that contact cells displaying hh protein at their surface are thereby instructed that they are in an appropriate location. Such a signal could provide a stimulatory, autocrine-type response. In contrast, if a cell that does not express $e n$ and does not have the hh protein on its surface contacts cells that do, it is thereby instructed that it is in an inappropriate location. Such a signal could lead to apoptosis and could help to explain why anterior and posterior cells do not mix.

\section{Materials and methods}

Fly culture and strains

Flies were maintained at $25^{\circ} \mathrm{C}$ on standard molasses medium. Wild type refers to an Oregon-R line. Enhancer trap lines that were screened for expression patterns were from the Spradling laboratory collection. Alleles $w g^{I G 22}, p t c^{I F 85}, n k d^{7 E}, h h^{I I 35}$ $h h^{9 D 94}, h h^{6 N 16}$, and $f t z^{W 20}$ were obtained from the Indiana Stock Center. en ${ }^{L A 1 O}$ (Kornberg 1981) has an ethylmethane sulfonate (EMS)-induced nonsense mutation at codon 305. hs-en3 (Poole and Kornberg 1988) carries a $\mathrm{P}$ transposon with a $h s p 70$ promoter-en cDNA fusion gene integrated in the second chromo- some. Stages are according to Campos-Ortega and Hartenstein (1985).

The P2023 enhancer trap line carries two transposons, located at $91 \mathrm{E} / \mathrm{F}$ and $94 \mathrm{D} / \mathrm{E}$, respectively. The transposons were separated by recombination. Only lines with the $94 \mathrm{D} / \mathrm{E}$ insertion expressed $\beta$-galactosidase in the posterior compartments, and one of these (P2023-44) was used for all experiments described in this study.

hs-en 3 embryos (2-3 hr after egg laying) were dechorionated with bleach and heat-shocked at $37^{\circ} \mathrm{C}$ for $30 \mathrm{~min}$. Embryos were processed for in situ hybridization or immunohistochemistry after incubation at $25^{\circ} \mathrm{C}$ for $3 \mathrm{hr}$.

\section{Detection of $\beta$-galactosidase with X-gal}

Imaginal discs were dissected from third-instar larvae in phosphate-buffered saline (PBS), fixed with ice-cold $0.2 \%$ glutaraldehyde in PBS, washed twice in PBS, and placed in X-gal (5-bromo-4-chloro-3-indolyl- $\beta$-D-galactopyranoside) staining solution $\left[0.2 \% \mathrm{X}\right.$-gal (diluted from an $8 \%$ stock in DMSO), $10 \mathrm{mM} \mathrm{NaPP}_{\mathrm{i}}$ (pH 7.0), $1 \mathrm{mM} \mathrm{MgCl}_{2}, 5 \mathrm{mM} \mathrm{K}_{4} \mathrm{Fe}(\mathrm{CN})_{6}$, and $\left.5 \mathrm{mM} \mathrm{K}_{3} \mathrm{Fe}(\mathrm{CN})_{6}\right]$. Discs were stained from 1 to $16 \mathrm{hr}$ at $37^{\circ} \mathrm{C}$, washed in staining solution that did not contain X-gal, and mounted in Fluoromount.

\section{Preparation and cloning of genomic DNA and isolation of RNA}

Genomic DNA for Southern analysis and cloning was prepared from 100 flies by use of a procedure based on Jowett (1986). Flies were homogenized in $2 \mathrm{ml}$ of $0.1 \mathrm{M}$ Tris (pH 9.0), $0.1 \mathrm{M}$ EDTA, $1.0 \%$ SDS, and $0.5 \%$ diethylpyrocarbonate (DEPC) and heated at $65^{\circ} \mathrm{C}$ for $30 \mathrm{~min}$. To this, $280 \mu \mathrm{l}$ of $8 \mathrm{M} \mathrm{KOAc}$ was added, and the mixture was chilled on ice for $30 \mathrm{~min}$ and clarified by centrifugation in a microcentrifuge at $4^{\circ} \mathrm{C}$ for $10 \mathrm{~min}$. DNA in the supernatant was precipitated with isopropanol and washed in $70 \%$ ethanol, dried, and resuspended in TE buffer $[10 \mathrm{mM}$ Tris (pH 7.5), 1 mM EDTA].

Poly $(A)^{+}$RNA was prepared from staged embryos and imaginal discs using the Fast Track mRNA isolation kit (Invitrogen). Southern and Northern analyses were as described in Sambrook et al. (1989). RNA standards were purchased from GIBCO-BRL. Reverse Northern analysis was performed by transcribing RNA isolated from imaginal discs or 0 - to 12 -hr embryos with reverse transcriptase, generating a probe representing the cDNA with random primers and DNA polymerase, and hybridizing to a Southern blot of 94D/E phage DNA. 


\section{Isolation and sequencing of hh cDNAs}

Genomic DNA adjacent to the P-element transposon in strain A937A (Bier et al. 1989) was cloned by plasmid rescue after digestion with EcoRI (Wilson et al. 1989). A 4-kb pMT21-derived plasmid was identified and its $2.1-\mathrm{kb}$ EcoRI-Bg/II fragment was used to isolate a genomic clone from a $\lambda$ phage library. After partial restriction mapping, phage with overlapping sequences were isolated, and the procedure was repeated twice to isolate $50 \mathrm{~kb}$ of contiguous sequence.

Several genomic fragments (Fig. 1A) were used to screen the 4to 8-hr embryonic cDNA library of Brown (Brown and Kafatos 1988). Four cDNA clones were isolated, and sequence was obtained from two of the largest clones, chh44 and chh46. Nested deletions of the $2.3-\mathrm{kb}$ chh 44 cDNA clone were generated by exonuclease digestion, and partial sequence $(>2 \mathrm{~kb})$ was obtained using the Sequenase DNA sequencing kit (U.S. Biochemical). Oligonucleotide primers were prepared for direct sequencing of both strands of the 2305 -bp chh46. The chh44 sequence obtained was identical to the chh46 sequence (Fig. 4), with the following differences: chh44 has an additional 49 bp at the 5 ' end; $\mathrm{C}$ at position $\mathrm{I}$; $\mathrm{G}$ at position 6; and an $\mathrm{A}$ at position 333 , giving a translation stop at codon 9 . Differences between chh46 and the genomic sequence determined by Mohler and Vani (1992) are the following: The genomic sequence has a $C$ at position 1, a $\mathrm{G}$ at position 6 , no nucleotide at position 98 , an $\mathrm{A}$ at position 105, and a $T$ at position 106.

Sequence comparisons were made with GenBank data bases using the FASTA program.

\section{In situ hybridization and antibody staining of whole-mount} embryos and imaginal discs

In situ hybridization to whole-mount embryos was performed according to Tautz and Pfeifle (1989), with modifications (Eaton and Kornberg 1990). For preparation of probe, the entire cDNA fragment of chh46 was gel isolated and labeled by the random priming method with digoxigenin-modified UTP.

Immunohistochemical detection of en protein was essentially as described in Karr et al. (1989). To detect en protein in embryos, ascites fluid containing the 4D9 mouse monoclonal antibody (Patel et al. 1989) was used at a dilution of $1: 100$. The secondary biotinylated donkey anti-mouse antibody (Jackson Immunologicals) was used at a dilution of $1: 1000$. Biotinylated antibody was detected with the Elite $\mathrm{ABC}$ kit and the DAB color reaction kit (Vectastain). For double detection of $h h$ mRNA and en protein, in situ hybridization was carried out, and embryos were processed for immunocytochemistry as described above except that the AEC color reaction kit (Vectastain) was used in place of the DAB kit.

\section{Acknowledgments}

We gratefully acknowledge Jym Mohler, John Lee, and Phil Beachy for generously sharing unpublished information. We also thank the Spradling and Jan laboratories for gifts of enhancer trap lines, the Jan laboratory for a $\lambda$ DASH (Stratagene) phage genomic library, Michael Weir for the wg cDNA, and Bill Theurkauf for an $\alpha$-tubulin clone. This work was supported by a postdoctoral fellowship from the Human Frontier Science Program to T.T., postdoctoral fellowships from the Jane Coffin Childs Memorial Fund and the California Division of the American Cancer Society to S.E., and grants from the National Institutes of Health to T.B.K.

The publication costs of this article were defrayed in part by payment of page charges. This article must therefore be hereby marked "advertisement" in accordance with 18 USC section 1734 solely to indicate this fact.

\section{References}

Baker, N.E. 1987. Molecular cloning of sequences from wingless, a segment polarity gene in Drosophila: The spatial distribution of a transcript in embryos. EMBO /. 6: 1765-1773.

Bier, E., H. Vaessin, S. Shephard, K. Lee, K. McCall, S. Barbel, L. Ackerman, R. Carretto, T. Uemura, E. Grell, L. Jan, and Y.N. Jan. 1989. Searching for pattern and mutation in the Drosophila genome with P-lacZ vector. Genes \& Dev. 3: 12731287.

Bopp, D., M. Murri, S. Baumgartner, G. Frigerio, and M. Noll. 1986. Conservation of a large protein domain in the segmentation gene paired and in functionally related genes of Drosophila. Cell 48: 745-755.

Brower, D. 1986. engrailed gene expression in Drosophila imaginal discs. EMBO I. 5: 2649-1656.

Brown, N.H. and F.C. Kafatos. 1988. Functional cDNA libraries from Drosophila embryos. J. Mol. Biol. 203: 425-437.

Campos-Ortega, J.A. and V. Hartenstein. 1985. The embryonic development of Drosophila melanogaster. Springer-Verlag, Berlin, Germany.

Condie, J.M. and D.L. Brower. 1989. Allelic interactions at the engrailed locus of Drosophila: engrailed protein expression in imaginal discs. Dev. Biol. 135: 31-42.

DiNardo, S., J. Kuner, J. Theis, and P.H. O'Farrell. 1985. Development of embryonic pattern in $D$. melanogaster as revealed by accumulation of the nuclear engrailed protein. Cell 43: 59-69.

Eaton, S. and T.B. Kornberg. 1990. Repression of $c i-D$ in posterior compartments of Drosophila by engrailed. Genes \& Dev. 4: 1068-1077.

Garcia-Bellido, A., P. Ripoll, and G. Morata. 1973. Developmental compartmentalizaton of the wing disc of Drosophila. Nat. New Biol. 245: 251-253.

Gilson, L., H.K. Mahanty, and R. Kolter. 1990 Genetic analysis of an MDR-like export system: The secretion of colicin $\mathrm{V}$. EMBO /. 9: 3875-3884.

Hama, C., Z. Ali, and T.B. Kornberg. 1990. Region-specific recombination and expression are directed by portions of the Drosophila engrailed promoter. Genes \& Dev. 4: 10791093.

Heemskerk, J., J. DiNardo, R. Kostriken, and P.H. O'Farrell. 1991. Multiple modes of engrailed regulation in the progres sion towards cell fate determination. Nature 352: 404-410.

Hidalgo, A. 1991. Interactions between segment polarity genes and the generation of the segmental pattern in Drosophila. Mech. Dev. 35: 77-87.

Hooper J.E. and M.P. Scott. 1989. The Drosophila patched gene encodes a putative membrane protein required for segmental patterning. Cell 59: 751-765.

Ingham, P.W., A.M. Taylor, and Y. Nakano. 1991. Role of the Drosophla patched gene in positional signalling. Nature 353: $184-187$.

Jaynes, J.B., and P.H. O'Farrell. 1988. Activation and repression of transcription by homeodomain-containing proteins that bind a common site. Nature 336: 744-749.

1991. Active repression of transcription by the Engrailed homeodomain protein. EMBO I. 10: 1427-1433.

Jowett, T. 1986. Preparation of nucleic acids. In Drosophila: $A$ practical approach (ed. D.B. Roberts), pp. 275-286. IRI Press, Oxford, England.

Jürgens, G. 1985. A group of genes controlling the spatial ex- 
pression of the bithorax complex in Drosophila. Nature 316: 153-155.

Karr, T., M.J. Weir, Z. Ali, and T. Kornberg. 1989. Patterns of engrailed protein in early Drosophila embryos. Development 105: 605-612.

Kinzler, K.W. and B. Voglestein. 1990 The GLI gene encodes a nuclear protein which binds sepcific sequences in the human genome. Mol. Cell. Biol. 10: 634-642.

Kornberg, T. 1981. engrailed: A gene controlling compartment and segment formation in Drosophila. Proc. Natl. Acad. Sci. 78: 1095-1099.

Komberg, T., I. Siden, P. O'Farrell, and M. Simon. 1985. The engrailed locus of Drosophila: In situ localization of transcripts reveals compartment specific expression. Cell 40: $45-53$.

Lawrence, P.A. and G. Morata. 1976. Compartments in the wing of Drosophila: A study of the engrailed gene. Dev. Biol. 50: $321-337$

Lawrence, P.A. and G. Struhl. 1982 Further studies on the engrailed phenotype in Drosophila. EMBO I. 1: 827-833

Martinez-Arias, A., N. Baker, and P. Ingham. 1988. Role of segment polarity genes in the definition and maintenance of cell states in the Drosophila embryo. Development 103: $157-170$.

McCrea, P.D., C.W. Turck, and B. Gumbiner. 1991. A homolog of the armadillo protein in Drosophila (plakoglobin) associated with E-cadherin. Science 254: 1259-1361.

Mohler, J. 1988. Requirements for hedgehog, a segment polarity gene, in patterning larval and adult cuticle of Drosophila. Genetics 120: 1061-1072.

Mohler, J. and K. Vani. 1992. Molecular organization and embryonic expression of the hedgehog gene involved in cell-cell communication in segmental patterning of Drosophila. Development 115: 957-971.

Mohler, J., N. Weiss, S. Murli, S. Mohammadi, K. Vani, G. Vasalakis, C.H. Song, A. Epstein, T. Kuang, J. English, and D. Cherda. 1992. The embryonically active gene unkempt, of Drosophila encodes a $\mathrm{Cys}_{3} \mathrm{His}$ finger protein. Genetics 131: 377-388.

Morata, G. and P.A. Lawrence. 1975. Control of compartment development by the engrailed gene of Drosophila. Nature 255: 614-618.

Nakano, Y.I., I. Guerrero, A. Hildago, A. Taylor, J. Whittle, and P. Ingham. 1989. A protein with several possible membrane spanning domains encoded by the Drosophila segment polarity gene patched. Nature 341: 508-513.

Nüsslein-Volhard, C. and E. Wieschaus. 1980. Mutations affecting segment number and polarity in Drosophila. Nature 287: 795-801.

Orenic, T.V., D.C. Slusarski, K.L. Kroll, and R.A. Holmgren. 1990. Cloning and characterization of the segment polarity gene cubitus interruptus dominant of Drosophila. Genes \& Dev. 4: 1053-1067.

Patel, N.H., E. Martin-Blanco, K.G. Coleman, S. Poole, M.C. Ellis, T.B. Kornberg, and C.S. Goodman. 1989. Expression of engrailed proteins in arthropods, annelids, and chordates. Cell 58: 955-968.

Peifer, M. and E. Wieschaus. 1990. The segment polarity gene armadillo encodes a functionally modular protein that is the Drosophila homolog of human plakoglobin. Cell 63: 11671178.

Poole, S.J. and T. Kornberg. 1988. Modifying expression of the engrailed gene of Drosophila melanogaster. Mechanisms of segmentation. Development (Suppl.) 104: 85-93.

Poole, S.J., L.M. Kauvar, B. Drees, and T. Komberg. 1985. The engrailed locus of Drosophila: Structural analysis of an em- bryonic transcript. Cell 40: 37-43.

Preat, T., P. Therond, C. Lamour-Isnaard, B. LimbourgBouchon, H. Tricare, I. Erk, M.-C. Mariol, and D. Busson. 1990. A putative serine/threonin kinase encoded by the segment polarity gene fused of Drosophila. Nature 347: 87-89.

Rijsewijk, F., M. Schuermann, E. Wagenaar, P. Parren, D. Weigel, and R. Nusse. 1987. The Drosophila homolog of the mouse mammary oncogene int 1 is identical to the segment polarity gene wingless. Cell 50: 649-657.

Robertson, H.M., C.R. Preston, R.W. Phillis, D.M. JohnsonSchlitz, W.K. Benz, and W.R. Engels. 1988. A stable genomic source of $P$ element transposase in Drosophila melanogaster. Genetics 118: 461-470.

Sambrook, J., E.F. Frisch, and T. Maniatis. 1989. Molecular cloning: A laboratory manual, 2nd ed. Cold Spring Harbor Laboratory Press, Cold Spring Harbor, New York.

Tautz, D. and C. Peifle. 1989 A non-radioactive in situ hybridization method for the localization of specific RNAs. Chromosoma 98: 81-85.

Weir, M. and T. Kornberg. 1985. Patterns of engrailed and fushi tarazu transcripts reveal novel intermediate stages in Drosophila segmentation. Nature 318: 433-439.

Wilson, C., R.K. Pearson, H.J. Bellen, C.J. O'Kane, U. Grossniklaus, and W.J. Gehring. 1989. P-element-mediated enhancer detection: An efficient method for isolating and characterizing developmentally regulated genes in Drosophila. Genes \& Dev. 3: 1301-1313. 


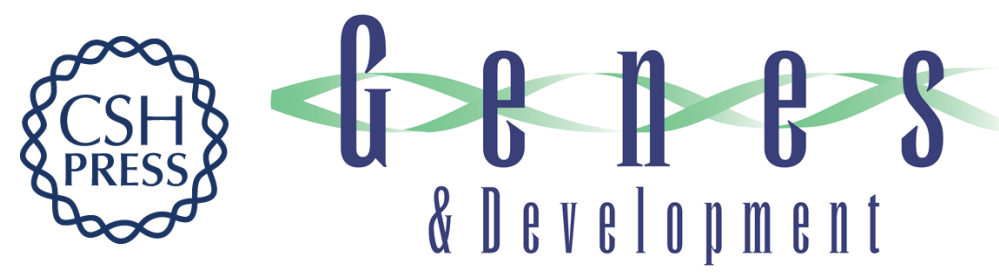

\section{The Drosophila hedgehog gene is expressed specifically in posterior compartment cells and is a target of engrailed regulation.}

T Tabata, S Eaton and T B Kornberg

Genes Dev. 1992, 6:

Access the most recent version at doi:10.1101/gad.6.12b.2635

References This article cites 43 articles, 13 of which can be accessed free at:

http://genesdev.cshlp.org/content/6/12b/2635.full.html\#ref-list-1

License

Email Alerting

Service

Receive free email alerts when new articles cite this article - sign up in the box at the top right corner of the article or click here.

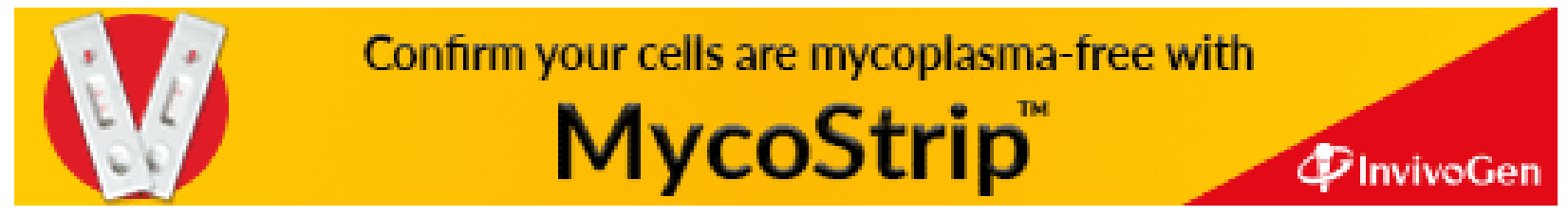

